\title{
Six basic rules to help doctoral students pave their way through $\mathrm{PhD}$ research
}

\author{
Raymond R. Bond \\ School of Computing, \\ Ulster University, UK \\ rb.bond@ulster.ac.uk
}

\begin{abstract}
This paper presents six simple guidelines or rules to help doctoral researchers in their PhD studies. The six rules could be used as part of an induction to a PhD programme or as a supplement to a research mentoring programme. The six rules are as follows: 1) research something that you are naturally interested in, 2) start where you are and set yourself up for progress, 3) know the landscape before deciding where to plant a tree, 4) do not be afraid of being a polymath and at least be a philomath, 5) focus on questions, not just answers, and 6) always think about the limitations and biases in your research. I also take the opportunity to share some of my favourite maxims and quotes to help support these rules.
\end{abstract}

Doctoral training, postgraduate education, PhD mentoring, PhD research, research methods.

\section{INTRODUCTION}

This paper summarises a plenary talk given at the 33rd International British Computer Society Doctoral Consortium for Human Computer Interaction. The paper presents guidelines and maxims that are relevant to all doctoral students regardless of their research discipline. The paper includes a series of personal reflections from my own academic experiences that have been condensed into six rules or guidelines for doctoral students. Of course, before outlining any maxims, 'no one should over trust or over-use a maxim'. A proverb, maxim, aphorism, rule or guideline is not always the best advice for every complex situation but having some overarching heuristics does help with decision making. These personal guidelines may help orientate students and researchers towards progressing through their research studies and career. There is no specific relevance regarding the order of the six rules.

\section{THE SIX RULES}

First Rule:

Research something that you are naturally interested in

It is integral to 'research something that you are naturally interested in', if indeed you are fortunate enough to have scope to manoeuvre a little in your studies. Somebody might ask you to research a 'difficult challenge', which is fine, providing that the challenge is naturally interesting to you. To quote
Ralph Waldo Emerson, "nothing great was ever achieved without enthusiasm" (Goodreads.com a). You are likely to engage more and achieve more when you are enthusiastic about what you are doing. Enthusiasm is likely to take you a little further and deeper into a subject. However, enthusiasm alone is, of course, not enough to achieve a PhD. Figure 1 provides a general framework or an aid to help you find your 'ideal research'. Being excited about the research is important, but ideally the research should also be challenging, and that challenge should be matched to your ability. Aligning your ability with the challenge is akin to the 'flow' concept defined by Csikszentmihalyi (2008), where a person is fully engaged and focussed during the flow state. As indicated in Figure 1, in addition to being challenging and engaging, the research question should be worth asking. In some cases, the question should have clear implications and potential for research impact.

\section{Second Rule: \\ Start where you are and set yourself up for progress}

Often you can feel overwhelmed when starting a $\mathrm{PhD}$, and you may concentrate on what you don't know as opposed to what you do know. I suppose this attitude can actually drive life-long learning that is driven by the never-ending feeling of not knowing enough. However, the feeling of not having the ability or knowledge to do a $\mathrm{PhD}$ is probably common. A quote from Theodore Roosevelt can often help during these times of 'imposter 
syndrome', "do what you can, with what you have, where you are" (Goodreads.com b). This kind of motivating wisdom can help us focus the mind on doing the best that we can do - with the knowledge and resources that we have. If the student does not have access to the best laboratories in the world, then they can only do their best based on the circumstances of what they have and where they are. Try to use your current knowledge and your profile as an advantage as opposed to seeing it as a disadvantage. For example, there are people with backgrounds in psychology doing PhDs in computer science, and these students can apply their knowledge of cognition to advance studies in human computer interaction or indeed Al research.

Students should focus on benchmarking themselves against themselves last week or indeed last month. As put by Jordan Peterson, "compare yourself to who you were yesterday, not to who someone else is today" (Peterson, 2018). You should avoid comparing your PhD with other PhDs. $\mathrm{A} P \mathrm{PhD}$ is a personal journey and no two PhDs are the same. It is good to compare your knowledge and progress with the knowledge and progress that you made in the preceding week. Benchmarking yourself against yourself yesterday maintains the focus on your own development and not on others. And the first paper you publish may not be the best paper you publish. According to Alain de Botton, "anyone who isn't embarrassed of who they were last year probably isn't learning enough" (Goodreads.com c). Taking a 'staircase approach' to your research is a good strategy, where you might publish an abstract and present your work at a conference before submitting a journal paper.

Very small steps in the right direction is still called 'progress'. A PhD is a major project requiring a number of years to complete. We know the Chinese proverb, "a journey of a thousand miles begins with a single step" (Phrases.org.uk). In academia, often looking at a major task can encourage procrastination, but starting with simple steps in the right direction can help start the journey, and continuing in this manner can help you finish it.

To make significant progress in your research career, it is prudent to develop relationships with a mentor or mentors. The latter is best since no one person can always give you the best possible advice for every situation. It is better to avail of a 'collective wisdom' from a group of mentors, some of which may provide seemingly contradictory advice, so learning to spit out the bones is also a skill worth acquiring. Having mentors allows us to learn from their mistakes as opposed to learning from our own. Good mentors also drive the best from you and help you achieve more than you thought you could. A good mentor will encourage you to think and reflect and give you the courage to define new concepts, neologisms and theories that prompt you to write much needed provocative papers. It is also important to realise that every single person has something to teach you - the skill is finding out what they know, that you don't. In addition to having mentors, developing a social network with other researchers who are within and outside your field of research is also important to engender interdisciplinary thinking and collaboration. And don't be too precious about sharing ideas with your network unless you have an interest in patents or other commercial programmes of work. When ideas are shared, they are stress tested by others and the ideas then evolve into better ones. In post-doctoral positions, the sharing of ideas is akin to planting seeds awaiting a harvest of research opportunities.

In relation to setting yourself up for progress, it is also important to be aware that "perfection is the enemy of progress" or "perfection is the enemy of the good enough" - a quote often attributed to Winston Churchill (Walkup, 2020). It is important to not overindulge in this maxim since the inverse can be that "good is the enemy of great" to quote Collins (2009). The main point of the former quote is that it is not productive or progressive to 'perfect' a less important task at the expense of starting more important tasks. For example, spending an inordinate amount of time on a literature review ensuring that all papers are included won't help progress the PhD if there isn't enough time to do your experiments and make a contribution to knowledge. Or indeed, you might spend too much time perfecting a thesis without ever getting to a viva. In some ways, this is the philosophy of agile software development where a minimum viable product is made available for testing allowing for refinement and rapid progress towards the end goal. The idea of developing a minimum viable product is that this imperfect prototype can help expedite the progress towards the end goal. It is important to avoid falling into the trap of perfectionism, unless perfectionism is necessary.

In relation to managing tasks, you might consider keeping in mind the Eisenhower matrix or indeed other variants (Bratterud, 2020). The Eisenhower matrix is a quadrant of the type of tasks that you may have based on the urgency and importance of the tasks. And it seems that people can prioritise tasks that are important and urgent and tasks that are urgent but not important, while perhaps ignoring tasks that are important but not urgent, for example, writing a research grant or a position paper might be important but not urgent. To progress, it is critical to prioritise the tasks that are important but not urgent. This tasks are likely to set you apart. This is the theory, but of course the advice is not always recommended for all circumstances. 


\section{Third Rule: \\ Know the landscape before deciding where to plant a tree}

Nobody wants to plant a tree (or themselves) in the wrong part of the garden. Not to push the analogy beyond its use, but once the tree takes root it can be difficult to uproot. I suppose this advice is relevant to anyone who is trying to decide what degree or career path to take. In research specifically, it is important to undertake a literature review before doing an experiment, otherwise your work may be ill-informed or indeed unnecessary. Hypotheses are often only credible when justified, supported and informed by work that has gone before.

It is crucial to look at your research discipline from a helicopter view in order to appreciate the ecosystem that the work fits into. That way you can develop a knowledge graph whilst being more creative with an understanding of the wider issues. This helicopter view also allows us to maximise the adoption or implications of the research. For example, many machine learning studies applied to medicine don't include co-authors who are medical experts which can result in studies with 'statistical significance' without having 'clinical significance'. Also, once you get too focussed on a niche area, you can experience cognitive tunnelling and you "can't see the forest for the trees" (Dictionary), meaning that you cannot see the bigger picture when fixated on a pixel. Engaging in the higher-level challenges of the discipline and having general conversations with multi-disciplinary researchers can help.

Barbara Oakley (2014) discusses the concepts of diffuse and focussed modes of thinking and learning. These concepts can be used to help researchers transition from concentrated thinking to generic (diffuse) thinking. Focussed learning or thinking is when we concentrate our attention on a research task or experiment perhaps at our desk or office. Whilst we focus, our mind is concentrated on the task at hand. However, diffuse learning is more about letting your mind wander whilst it conjures-up new ideas by connecting your focussed knowledge with other existing concepts in your mind to assist with problem solving or to invent new disciplines or applications. Diffuse thinking can be engendered through activities away from the office or by engaging in unrelated activities. For example, Thomas Edison apparently scheduled naps, perhaps to encourage the toggling between focussed and diffuse modes of thinking (Oakley, 2014). Although I am not saying that you should sleep at work but perhaps going for a walk is a good approach to encourage diffuse thinking. Indeed, people can spark innovative ideas during a walk, a shower or unexpectedly conceive solutions during an unrelated activity. Perhaps this is due to our subconsciousness working through problems during these diffuse states and subsequently mailing the headlines to our conscious mind (Eagleman, 2013).

I suppose a closely related concept to mind wandering is the use of imagination, Albert Einstein said "imagination is more important than knowledge. For knowledge is limited, whereas imagination embraces the entire world, stimulating progress, giving birth to evolution. It is, strictly speaking, a real factor in scientific research" (Goodreads.com d).

\section{Fourth Rule: \\ Do not be afraid of being a polymath and at least be a philomath}

Of course, focusing on a specific research problem helps progress science given the depth that a concentrated piece of research can provide. However, engaging and reading in other fields can help provide new insights and innovations to your primary area of work. Creative research often comes by fusing fields or ideas together. It has been said that one can be 'a jack of all trades, and a master of none' (often attributed to Benjamin Franklin), but other variants of this quote have additional wisdom, including "a Jack of all trades and a master of one" or "a Jack of all trades and a master of some". Others report that the original quote was "a jack of all trades is a master of none, but oftentimes better than a master of one" (Quora). This original version of the quote is more like a complement to those who are multi-skilled as opposed to a criticism. According to Simmons (Medium.com, 2018), "if being a generalist was the path to mediocrity, why did the most comprehensive study of the most significant scientists in all of history uncover that 15 of the 20 were polymaths? Newton. Galileo. Aristotle. Kepler. Descartes. Huygens. Laplace. Faraday. Pasteur. Ptolemy. Hooke. Leibniz. Euler. Darwin. Maxwellall polymaths". It could be argued that it is easier (but maybe more innovative) to be mediocre in a few fields than it is to be a world expert in one field. And of course, not everyone can play in the premier league of quantum physics. Many researchers might consider a ' $T$ ' structure to their research interests, where there is a horizontal breadth across many domains with a vertical depth to one domain. Those who transcend the boundary of their core research expertise have the advantage of being agile and adaptive in terms of undertaking research when important multidisciplinary opportunities arise. A particular example at the time of writing this paper, is the current demand for research related to COVID-19, which has arguably been addressed by many agile and adaptive researchers. A quote that is often attributed 
(possibly misattributed) to either Charles Darwin or Leon C. Megginson, "it is not the strongest or the most intelligent who will survive but those who can best manage change" (Goodread.com e). And another quote, attributed to Alvin Toffler, makes the same point - "the illiterate of the 21st century will not be those who cannot read and write, but those who cannot learn, unlearn, and relearn" (Goodreads.com f).

If you are not interested in multidisciplinary research or polymaths, it is important to at least be a philomath, which means to be a lover of learning. This should hopefully be a natural characteristic of any $\mathrm{PhD}$ researcher. Of course, it takes a little reflection and introspection to identify the topics that you 'love' learning about. One exercise or activity that I did recently (which I recommend to you) is to place the last 10 or 20 books that you have read on the floor and cluster them into groups. Then label the clusters based on the topics that emerge. This also works by clustering the books or papers that inspired you during your lifetime. This simple exercise helps you to introspect and to get a sense of what topics you consistently enjoy reading about. For example, you might discover that you enjoy reading about fields like cognitive science and statistics and then realise that those fields could be combined, for example, to study cognitive bias in statistical analysis and visualisation. These types of activities are important to help us get to know ourselves. This exercise can also help to generate new ideas for post-doctoral research or to define new projects for your own prospective PhD students.

\section{Fifth Rule:}

\section{Focus on questions, not just answers}

This guideline is to encourage you to start your $\mathrm{PhD}$ 'focusing on questions as opposed to answers'. A significant part of science and discovery is asking the right questions. This is obvious but I find that we don't spend nearly enough time exploring hypotheses or the research questions that we could ask. Of course, questions need to be assessed for their inherent quality, e.g. can the question be answered by scientific experimentation? Can the hypothesis be falsified? And what would you measure and how would you measure it? More importantly, is the research question worth answering? Would the answer provide the discipline with useful knowledge and have implications? However, forgetting these inevitabilities, students and researchers should practice the art of asking questions. To me, it seems that as infants, we perpetually ask questions to the annoyance of our parents, but very quickly we stop 'asking' perhaps because our pedagogical experiences involve training on how to answer questions and little about how to compose questions. According to Confucius, "the 'person' who asks a question is a fool for a minute, the 'person' who does not ask is a fool for life" (Goodreads.com g). The word 'man' in the original quote has been replaced with 'person'.

It is good practice for students to maintain a log of research questions and counterfactuals so that they can practice and develop the habit of 'asking' research questions. Eventually, the researcher will hopefully discover a golden question that is worth answering, if indeed the question can be answered within a resource limited $\mathrm{PhD}$ programme. Practicing the art of asking questions is akin to running a marathon - a person doesn't wake up and run 26 miles, but runners practice profusely before having the ability to run a marathon. And perhaps practicing and asking lots of mediocre questions is a necessary precursor before composing a useful research question. Training on the art and science of asking questions should perhaps be a doctoral training course in every university. In agreement with Vale (2013), we need more "inquiry-based learning and less fact-based memorization" in our courses. For example, in my own PhD, I spent too much of my time developing data processing and visualisation software whilst often forgetting about the importance of asking the right questions. I will summarise this rule with a quote attributed to Pierre-Marc-Gaston de Lévis, "It is easier to judge the mind of a 'person' by 'their' questions rather than 'their' answers" (Wikipedia). A similar quote is attributed to Voltaire, "judge a 'person' by 'their' questions rather than by 'their' answers" (Goodreads.com h). The words 'man' and 'his' in the original quotes has been replaced here with the words 'person' and 'their' respectively.

\section{Sixth Rule: \\ Always think about the limitations and biases in your research}

This last rule is certainly not the least important. It is critical to know and debate the limitations of your research methods and findings. This can be aided by developing a working knowledge of the philosophy of science which is a field that interrogates the limitations of science and the scientific method, as well as the exploration of how science should ideally be carried out. For example, it is already well circulated that correlation is not necessarily causation, yet researchers often ask questions concerning 'cause and effect' but answer it with a study that measures association/correlation. Knowing the scientific method and concepts such as falsification, induction and deduction are helpful to understanding the limitations of your work. Understanding pseudoscience (fake science) can also help you build an understanding of what science looks like and what it doesn't look like (this is known as the 'demarcation problem' in science). Books such as 'Bad Science' authored by Ben 
Goldacre (Goldacre, 2010) are excellent for providing an overview on what good and bad science is.

Interrogating the limitations of your own research promotes research integrity and helps you articulate these limitations in any paper or thesis that you write. Knowing the principle of falsification is also important, as according to Einstein, "no amount of experimentation can ever prove me right; a single experiment can prove me wrong" (Goodreads.com i). This explains why experiments and hypothesis testing are typically set up to falsify a hypothesis. Moreover, many people will use induction or deduction methods or indeed a combination of both when doing research. There are always limitations to such methods, for example induction alone might tell you that all swans are white because all swans in your sample of observations are white, which sounds reasonable, but in fact, black swans do exist. This highlights the problem of generalisation from an experiment or theory that is supported by induction alone. Bertrand Russell also illustrates the limitation of induction using a story about the inductivist turkey living on a farm (Chalmers, 2013). In summary, the turkey records a lot of observations that it was fed at 9am each morning. The turkey made the same observation under many different circumstances and for many days, and 'inductively' concluded that it would continue to be fed at 9am on each prospective morning. However, Christmas morning came, and you can imagine what happened next (the end of the turkey). Knowing the limitations can help you develop more rigorous and more scientific experiments later on in your $\mathrm{PhD}$, or after your $\mathrm{PhD}$. There are always limitations to research, whether it be the convenience sampling, the sample size, the lack of confounding factors that were measured, the analysis (e.g. the unwarranted removal of outliers), the study design and so on. Many studies lack a control group or a placebo group because it is more convenient to record and compare pre- and post-intervention measures which of course are biased in many ways, including phenomena such as 'regression to the mean' and the 'placebo effect'. For example, regression to the mean is simply the fact that people who perform poorly in a test will likely do better in the next test, or indeed those who excel in a test will likely not do just as well in a subsequent test (hence they are regressing to the mean). Today, a major limitation to many studies is that they are not reproducible because of a lack of detail that is presented in the methods section or for some other statistical reason. Some argue that this has brought about a reproducibility crisis (Baker, 2016).

Understanding bias in research is key to understanding and acknowledging some of the limitations. There are numerous cognitive biases that we should all be made aware of, including anchoring, priming and the availability bias to name but a few, most of which can be read in detail in the scholarly work of Kahneman (2011), a noble prize winner. A well-known cognitive bias is of course 'confirmation bias', where even researchers can at times select, read and cite articles that they agree with, and ignore works that contradict their hunches. The book 'Incognito' by David Eagleman convinces us that our brain knows a lot more than we do, pointing to the fact that the conscious part of the brain is not always exposed to the hidden pattern recognisers, automatic processes or implicit knowledge that we use every day for efficient but often flawed decision making. There are many examples of bias in research practice and study designs. Some types of bias are more widely known than others. For example, the 'Hawthorne effect' (Wickström, 2000) is a well-known bias where subjects can change their behaviour just by being observed as part of a research study, where removing the researcher seems to remove the effect. This can mean that the cause is the act of observing subjects and not the intervention itself. This also reminds me of Goodhart's law (Chrystal, 2003) which tells us that once you make a metric a target or KPI ('Key Performance Indicator'), that metric may no longer measure what you think it does. This is because people or organisations game play to achieve the KPI, metric or benchmark, and thus it is no longer as useful as it was once thought to be. For example, a famous example included a bounty scheme that was created to reduce the number of rats in the community (Vann, 2003), and so people were asked to hand over rat tails as evidence that they killed some rats. The scheme was counterproductive since rats had been observed running around with no tails, and perhaps some people would have likely coordinated the reproduction of rats in order to collect more rat tails. Sampling bias, and in particular convenience sampling is another common limitation or bias in research. We often recruit subjects from our own university or region hence the cohort can often be homogeneous and unrepresentative of the wider population. These types of biases are obvious, but they are not always discussed in research papers. Indeed, analysing data can also include biases if researchers exclude outliers or unknowingly engage in 'p hacking' which is analysing the data in many ways until the inferential $p$-value is $<0.05$. Many studies also use a multitude of statistical tests on the same dataset without any Bonferroni corrections which can increase the likelihood of false discoveries. This is perhaps in part facilitated by the so-called 'cliff effect' and dichotomous thinking from interpreting statistical tests and pvalues (Rosenthal, 1963). The cliff effect is when researchers fail to see the $p$-value as a continuous number/statistic, and simply treat it as a binary 
statistic, i.e. they interpret it as either being less than $5 \%$ or more than $5 \%$ with no 'fuzzy logic'. Another common bias in research more generally, is the file drawer effect, where researchers and peer reviewers may be biased in submitting/publishing/accepting research that has a positive result (where $p<0.05$ ). This can mean that the body of published work is missing the unpublished studies that didn't find an effect. There is always a chance that a study can achieve a false positive result and even those studies are more likely to be published. Therefore, the worst case is that only the false positive and true positive studies get published and not the true negatives and false negatives. In other words, there can be a bias towards publishing false positive studies over true negatives. Of course, a priori registrations of a research study or trial do exist to try and manage this problem.

Finally, within this rule, it is opportune to mention that computer science research has a number of its own biases to be aware of. For example, in machine learning, there can be a 'class bias', where the algorithm is over exposed to one type of class, hence the algorithm might be biased towards that class in making its predictions. Also algorithms may not generalise well or indeed exhibit unintended gender or racial discrimination in its predictions. Computer science students may wish to read more about bias in machine learning in the references provided (O'Neil, 2016) (Bond et al., 2019). And $\mathrm{HCl}$ researchers should be aware that there are many user experience research opportunities to combat these problems, for example through researching and building explainable user interfaces and $\mathrm{HCl}$ enhanced decision support tools (Cairns et al., 2016).

\section{CONCLUDING REMARKS}

A seventh rule or bonus rule might be to develop resilience as a $\mathrm{PhD}$ researcher. Experiencing examinations from other senior academics or a peer reviewer can engender feelings of disappointment but learning from this experience or learning to articulate your response with humility is important. Also, don't be too overwhelmed by all the questions being asked during your PhD - not all questions are easily answered. According to one English proverb, "a fool can ask more questions in an hour than a wise 'person' can answer in seven years" (as before the word 'man' in the quote was replaced with the word 'person'). Put differently, asking critical research questions about your research is often easier than answering them. In terms of resilience, I recently read a story about Adam Cheyer (Vlahos, 2019). Adam as a young boy tried to join a computer club but was told that he could not join because he wasn't a computer programmer, and was informed that "it's not a club. It's a team" (Vlahos, 2019). Being resilient, Adam collected the materials from the trash and undertook the exercises on his own. Fast forwarding to many years later, Adam Cheyer was instrumental to the development of Siri - a well known intelligent voice assistant that has been built into Apple products. Being resilient is clearly important for making progress. I also recently picked up an important sentence in Stuart Russell's book (Russell, 2019), "There is a difference between climbing Everest and being deposited on top by a helicopter". This excerpt helps us to recognise that there is often greater meaning in difficult journeys. For me, the quote highlights the fact that the resilient person with the difficult journey may experience Everest in a very different way that is more satisfying and awe inspiring. Finally, to conclude with a general piece of advice engendering positivity with yourself and that from others, staying humble and avoiding the habit of stubbornness (because none of us know that much, really) is generally good counsel to researchers. And of course, "Be less curious about people and more curious about ideas" (quote attributed to Marie Curie) (Goodreads.com j). 
It is a research

question worth

answering
There are clear implications that would inform products, policy, technology,

understanding etc.

\section{You are excited about it}

\section{Your ideal research}

You have the ability

\author{
It is reasonably \\ challenging to you
}

Figure 1: Factors relevant to a student's ideal research agenda.

\section{REFERENCES}

Baker, M., 2016. Reproducibility crisis. Nature, 533(26), pp.353-66.

Bond, R., Mulvenna, M.D., Wan, H., Finlay, D.D., Wong, A., Koene, A., Brisk, R., Boger, J. and Adel, T., 2019, August. Human Centered Artificial Intelligence: Weaving UX into Algorithmic Decision Making. In RoCHI (pp. 2-9).

Bratterud, H., Burgess, M., Fasy, B.T., Millman, D.L., Oster, T. and Sung, E.C., 2020, August. The Sung Diagram: Revitalizing the Eisenhower Matrix. In International Conference on Theory and Application of Diagrams (pp. 498-502). Springer, Cham.

Cairns, A.W., Bond, R.R., Finlay, D.D., Breen, C., Guldenring, D., Gaffney, R., Gallagher, A.G., Peace, A.J. and Henn, P., 2016. A computerhuman interaction model to improve the diagnostic accuracy and clinical decision-making during 12-lead electrocardiogram interpretation. Journal of biomedical informatics, 64, pp.93-107.

Chalmers, A.F., 2013. What is this thing called science?. Hackett Publishing.

Chrystal, K.A., Mizen, P.D. and Mizen, P.D., 2003. Goodhart's Law: its origins, meaning and implications for monetary policy. Central banking, monetary theory and practice: Essays in honour of Charles Goodhart, 1, pp.221-243.

Collins, J., 2009. Good to Great-(Why some companies make the leap and others don't). William Collins.
Csikszentmihalyi, M., 2008. Flow: The psychology of optimal experience. Harper Perennial Modern Classics.

Dictionary.com, Can't see the forest for the trees | Definition of Can't see the forest for the trees at Dictionary.com, Available at: https://www.dictionary.com/browse/can-t-seethe-forest-for-the-trees [Accessed September 21, 2020].

Eagleman, D., 2013. Incognito. Robert Laffont.

Goldacre, B., 2010. Bad science: Quacks, hacks, and big pharma flacks. McClelland \& Stewart.

Goodreads.com a, Quote by Ralph Waldo Emerson: "Enthusiasm is one of the most powerful engines ..." Available at: https://www.goodreads.com/quotes/294967enthusiasm-is-one-of-the-most-powerfulengines-of-success [Accessed September 21, 2020].

Goodreads.com b, Quote by Theodore Roosevelt: "Do what you can, with what you have, where you ..." Available at: https://www.goodreads.com/quotes/188-dowhat-you-can-with-what-you-have-where-you [Accessed September 21, 2020].

Goodreads.com c, Quote by Alain de Botton: "Anyone who isn't embarrassed of who they were I..." Available at: https://www.goodreads.com/quotes/707150anyone-who-isn-t-embarrassed-of-who-theywere-last-year [Accessed September 21, 2020]. 
Goodreads.com d, Quote by Albert Einstein: "I believe in intuition and inspiration. Imagina..." Available at: https://www.goodreads.com/quotes/423568-ibelieve-in-intuition-and-inspiration-imaginationis-more-important [Accessed September 21, 2020].

Goodreads.com e, Quote by Leon C. Megginson: "It is not the strongest or the most intelligent..."Available at: https://www.goodreads.com/quotes/293400-it-isnot-the-strongest-or-the-most-intelligent-who [Accessed September 21, 2020].

Goodreads.com f, Quote by Alvin Toffler: "The illiterate of the 21st century will not be ..."Available at: https://www.goodreads.com/quotes/8800-theilliterate-of-the-21st-century-will-not-be-those [Accessed September 21, 2020].

Goodreads.com g, Quote by Confucius: "The man who asks a question is a fool for a min..." Available at: https://www.goodreads.com/quotes/184310-theman-who-asks-a-question-is-a-fool-for [Accessed September 21, 2020].

Goodreads.com h, Quote by Voltaire: "Judge a man by his questions rather than by his..." Available at: https://www.goodreads.com/quotes/6885-judgea-man-by-his-questions-rather-than-by-his [Accessed September 21, 2020].

Goodreads.com i, Quote by Albert Einstein: "No amount of experimentation can ever prove me ..." Available at: https://www.goodreads.com/quotes/51573-noamount-of-experimentation-can-ever-prove-meright-a [Accessed September 21, 2020].

Goodreads.com j, Quote by Marie Curie: "Be less curious about people and more curious a..." Available https://www.goodreads.com/quotes/45949-beless-curious-about-people-and-more-curiousabout-ideas [Accessed September 21, 2020].

Kahneman, D., 2011. Thinking, fast and slow. Macmillan.

Medium.com, People Who Have "Too Many Interests" Are More Likely To Be Successful According To Research | by Michael Simmons | Accelerated Intelligence | Medium, Available at: https://medium.com/acceleratedintelligence/modern-polymath81f882ce52db [Accessed September 21, 2020].

Oakley, B., 2014. A Mind for Numbers: How to Excel at Math and Science. Tarcher.
O'Neil, C., 2016. Weapons of math destruction: How big data increases inequality and threatens democracy. Broadway Books.

Peterson, J.B., 2018. 12 rules for life: An antidote to chaos. Penguin Random House Canada.

Phrases.org.uk, A journey of a thousand miles begins with a single step' - meaning and origin. Available at: https://www.phrases.org.uk/meanings/a-journeyof-a-thousand-miles-begins-with-a-singlestep.html [Accessed September 21, 2020].

Quora.com, Charlene Dargay's answer to What is the origin of the phrase Jack of All Trades Master of None; and what does it mean? Quora, Available at: https://www.quora.com/What-is-the-origin-of-thephrase-Jack-of-All-Trades-Master-of-None-andwhat-does-it-mean/answer/Charlene-

Dargay [Accessed September 21, 2020].

Rosenthal, R. and Gaito, J., 1963. The interpretation of levels of significance by psychological researchers. The Journal of Psychology, 55(1), pp.33-38.

Russell, S., 2019. Human compatible: Artificial intelligence and the problem of control. Penguin.

Vale, R.D., 2013. The value of asking questions. Molecular biology of the cell, 24(6), pp.680-682.

Vlahos, J., 2019. Talk to Me: How Voice Computing Will Transform the Way We Live, Work, and Think. Houghton Mifflin Harcourt.

Vann, M.G., 2003. Of rats, rice, and race: The great Hanoi rat massacre, an episode in French colonial history. French Colonial History, 4(1), pp.191-203.

Walkup, J.T. and Strawn, J.R., 2020. High-quality antidepressant prescribing: please consider whether "perfection is the enemy of progress". BMC Medicine, 18(1), pp.1-3.

Wickström, G. and Bendix, T., 2000. The" Hawthorne effect"-what did the original Hawthorne studies actually show?. Scandinavian journal of work, environment \& health, pp.363367.

Wikipedia, Pierre Marc Gaston de Lévis, Duke of Lévis - Wikipedia, Available at: https://en.wikipedia.org/wiki/Pierre_Marc_Gaston _de_L\%C3\%A9vis,_Duke_of_L\% $\%$ C $\%$ A9vis [Ac cessed September $21,20 \overline{2} 0]$. 